\title{
Effect of Indinavir on the Intestinal Exsorption of Amprenavir, Saquinavir and Nelfinavir after Intravenous Administration in Rats
}

\author{
Weihua Gao, Michiharu Kageyama, Yuji Inoue, Jun Tadano, Kyoko Fukumoto, Keizou Fukushima, \\ Daisuke Yamasaki, Asako Nishimura, Yukako Yoshikawa, Nobuhito Shibata, ${ }^{*}$ and Kanji TakadA \\ Department of Pharmacokinetics, Kyoto Pharmaceutical University; 5 Nakauchi-cho, Misasagi, Yamashina-ku, Kyoto \\ 607-8414, Japan. Received July 5, 2002; accepted November 7, 2002
}

To elucidate drug interaction between human immunodeficiency virus (HIV) protease inhibitors (PIs), the effect of indinavir (IDV) on the intestinal exsorption of other HIV PIs, amprenavir (APV), saquinavir (SQV) and nelfinavir (NFV) was investigated in rats using an in situ single perfusion method. IDV in the intestinal perfusate inhibited the exsorption of rhodamine 123 (Rho123), a known P-glycoprotein (P-gp) substrate, from blood into intestinal lumen in a concentration-dependent manner, and the inhibitory potency of $10 \mu_{\mathrm{M}} \mathrm{IDV}$ in the perfusate was close to that of $10 \mu \mathrm{M}$ cyclosporin A (CsA) in the perfusate. Ten $\mu \mathrm{M}$ of IDV in the intestinal perfusate also decreased significantly the exsorption clearance of Rho123 after intravenous administration. The IDV concentration in this system was not likely to cause hepatic interaction between HIV PIs, because the plasma IDV concentration was far below its inhibition constants for other HIV PIs in the liver microsomes. Thus, $10 \mu \mathrm{M}$ of IDV was chosen to investigate the effect of this inhibition on the exsorption of APV, SQV and NFV. IDV in the intestinal perfusate markedly increased the exsorbed amounts of SQV and NFV but not APV after intravenous administrations. Their exsorption clearances, however, showed only a slight increasing tendency or remained unchanged. These findings suggest that in addition to P-gp inhibition, other factors such as CYP3A inhibition might be important in the drug interaction of IDV with APV, SQV and NFV after intravenous administration in rat small intestine. The results obtained in this study will provide useful information to discuss the interactions among PIs when a double protease therapy is used for in HIV-infected patients.

Key words indinavir; intestinal exsorption; P-glycoprotein; amprenavir; saquinavir; nelfinavir

Double protease therapy, treatment with two kinds of human immunodeficiency virus (HIV) protease inhibitors (PIs), has recently been advocated clinically in the treatment of HIV-infected patients because of poor compliance, side effects and viral resistance in the highly active anti-retroviral therapy (HAART). ${ }^{1,2)}$ The HIV PIs, indinavir (IDV), amprenavir (APV), saquinavir (SQV), nelfinavir (NFV) and ritonavir (RTV), have been commonly used in this double protease therapy. ${ }^{3)}$ The drug interaction among these PIs, however, might occur with high frequency and complexity, because all the PIs are metabolized via CYP3A and are substrates and/or inhibitors of the membrane efflux transporter, P-glycoprotein (P-gp). ${ }^{4,5)}$ To select an optimal combination of HIV PIs with little drug interaction, therefore, remains one of the major tasks in clinical practice. ${ }^{6}$

IDV is a potent and highly selective inhibitor of HIV protease and also a substrate of the CYP3A and P-gp systems in rats and humans as well as in vitro systems. ${ }^{7-9)}$ According to the previous studies, the degrees of in vivo pharmacokinetic interaction between any combination of two drugs selected out of the above five were highly variable. ${ }^{10-12)}$ Even though IDV had the strongest inhibitory effect on APV metabolism in rat liver microsomes, the in vivo effects of an HIV PI after co-administration with IDV could not always be predicted from in vitro results, suggesting the presence of another interaction process besides metabolism in the liver. ${ }^{13)}$ It has been suggested that in some drugs the role of intestinal metabolism is greater than that of hepatic metabolism in the overall first-pass effect. ${ }^{14-16)}$

P-gp acts as an active secretion system or an absorption barrier by transporting a wide variety of structurally diverse compounds from the intestinal cells into the lumen, ${ }^{17-19)}$ and is known to be co-expressed with the CYP3A system in or- gans such as the intestine and liver. ${ }^{20)}$ Moreover, P-gp and CYP3A share many substrates and inhibitors, including the four PIs, IDV, APV, SQV and NFV. ${ }^{6,21,22)}$ In addition, our study using a microdialysis method suggested that potential drug interactions between IDV and the three other HIV PIs, APV, SQV and NFV occurred in both the liver and intestine, and the degree varied in every combination with IDV. ${ }^{23}$ The contribution of intestinal exsorption via P-gp on the drug interactions between two PIs is, however, still unclear.

In the present study, focusing on the intestinal exsorption of PIs, we investigated the effect of IDV on the exsorption of APV, SQV and NFV via the P-gp-related transport from blood to the intestine using an in situ single perfusion method.

\section{MATERIALS AND METHODS}

Chemicals IDV, APV and SQV were kindly supplied by Vertex Pharmaceuticals Inc. (Cambridge, MA, U.S.A.), Merck Sharp, Dohme Research Laboratories (Rahway, NJ, U.S.A.) and Hoffman-LaRoche Inc. (Nutley, NJ, U.S.A.), respectively. NFV was extracted from commercial tablets and the crude extract was purified by a preparative HPLC method. The chemical structures of the four PIs are shown in Fig. 1. Rhodamine 123 (Rho123) and cyclosporin A (CsA) were purchased from Sigma Chemical Co. (Steinhein, Germany). Acetonitrile (HPLC grade) and diethyl ether were obtained from Kanto Chemical Co. Inc. (Tokyo, Japan). All other reagents used were of analytical grade and were used without further purification.

Preparation of Standard and Test Solutions The standard stock solutions of the four HIV PIs were prepared by dissolving them in ethanol at various concentrations and 


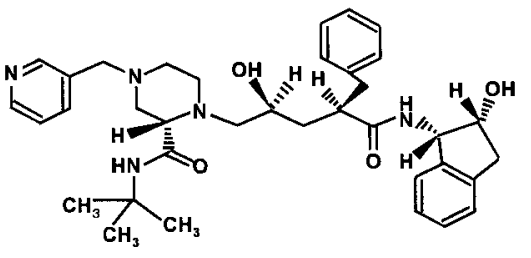

Indinavir (IDV)

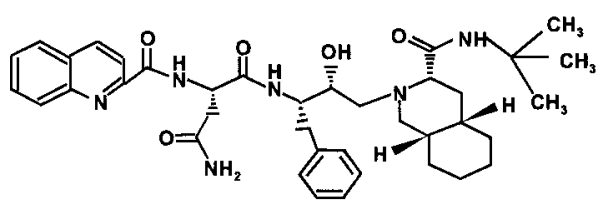

Saquinavir (SQV)

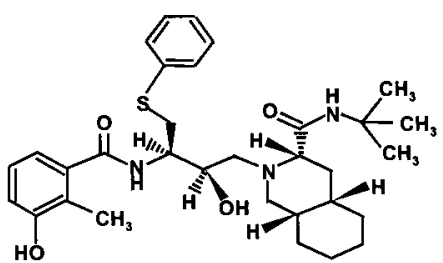

Nelfinavir (NFV)

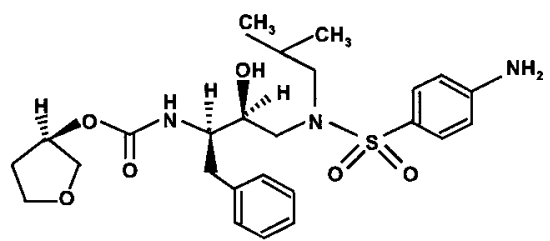

Amprenavir (APV)

Fig. 1. Chemical Structures of HIV PIs Tested

were stored at $4{ }^{\circ} \mathrm{C}$ in the dark. Known amounts of these standard stock solutions were added to plasma or perfusate in a volume ratio of $1: 100$ to prepare samples of the calibration curve. Rho123 was dissolved at a concentration of $100 \mu \mathrm{M}$ in Dulbecco's phosphate-buffered saline (D-PBS) containing $5 \%$ mannitol. For the intravenous administration, APV, SQV and NFV were each dissolved at a concentration of $5 \mathrm{mg} / \mathrm{ml}$ in a vehicle composed of $5 \%$ ethanol, $5 \%$ polyoxyethylene (40) hydrogenated castor oil (HCO-40) and 5\% Pharmasolve ${ }^{\circledR}$ in distilled water. The perfusate for in situ intestinal perfusion was composed of $25 \mathrm{~mm}$ glucose and $5 \%$ ethanol in DPBS with $0.1,1,10 \mu \mathrm{M}$ IDV or $10 \mu \mathrm{M}$ CsA or without drugs as control.

Animals Male Wistar rats, weighing approximately 280 to $320 \mathrm{~g}$ were obtained from Nippon SLC Co., Ltd. (SLC, Hamamatsu, Japan). Rats had free access to food and water and were maintained in a temperature-controlled facility with a 12-h light/dark cycle for at least one week before use. All animal experiments were carried out in accordance with the Guidelines for Animal Experimentation in Kyoto Pharmaceutical University.

Preparation of Intestinal Microsome and Enzyme Kinetic Analysis Preparation of rat intestinal microsomes was performed according to the method of Fasco et al. with some modifications. $\left.{ }^{24}\right)$ Rat intestine $(60 \mathrm{~cm}$ from the pyloric region) was excised quickly under light anesthesia with ether, and flushed with $50 \mathrm{ml}$ of solution A $(1.5 \mathrm{~mm} \mathrm{KCl}, 96 \mathrm{~mm}$ $\mathrm{NaCl}, 27 \mathrm{~mm}$ sodium citrate dihydrate, $8 \mathrm{~mm} \mathrm{KH}_{2} \mathrm{PO}_{4}$, $5.6 \mathrm{~mm} \mathrm{Na} \mathrm{HPO}_{4}, 0.23 \mathrm{~mm}$ phenylmethyl sulponyl fluoride (PMSF), $\mathrm{pH} 7.3$ ) on an ice-cooled petri dish. The proximal end of the intestinal segment was then clamped and the intestine was filled with solution A, clamped at the distal end, and incubated at $37^{\circ} \mathrm{C}$ for $15 \mathrm{~min}$ in $100 \mathrm{ml}$ of this solution. The solution in the lumen was then drained from the distal end and replaced with $15 \mathrm{ml}$ of solution $\mathrm{B}(136.9 \mathrm{mM} \mathrm{NaCl}$, $2.68 \mathrm{mM} \mathrm{KCl}, 1.47 \mathrm{~mm} \mathrm{KH} \mathrm{PO}_{4}, 8.1 \mathrm{~mm} \mathrm{Na} \mathrm{HPO}_{4}, 1.5 \mathrm{~mm}$ EDTA, $0.5 \mathrm{~mm}$ ditiothreitol, $0.23 \mathrm{~mm}$ PMSF, pH 7.2). After peeling the epithelial cells off the intestine gently by hand, the clouded solution was collected into a clean centrifuging tube. This process was repeated three times. After centrifuging $\left(800 \times \boldsymbol{g}, 4^{\circ} \mathrm{C}\right)$ for $10 \mathrm{~min}$ and removing the supernatant, the pellet was washed twice with $20 \mathrm{ml}$ of solution $\mathrm{C}(5 \mathrm{~mm}$ histidine, $0.25 \mathrm{M}$ sucrose, $0.5 \mathrm{~mm}$ EDTA and $0.23 \mathrm{~mm}$ PMSF, $\mathrm{pH}$ 7.3). The epithelial cells were homogenized in $5 \mathrm{ml}$ of solution $\mathrm{C}$ in a glass-Teflon homogenizer kept on ice. The homogenates were centrifuged at $4^{\circ} \mathrm{C}$ for $15 \mathrm{~min}$ at $15000 \times \boldsymbol{g}$ and the supernatant was centrifuged again at $105000 \times \boldsymbol{g}$ for $65 \mathrm{~min}$. Finally, microsomal pellets were resuspended in solution D $(0.1 \mathrm{M}$ Tris, $10 \mathrm{~mm}$ EDTA $)$ to get a concentration of $4.0 \mathrm{mg}$ protein $/ \mathrm{ml}$. Microsomal protein concentration was determined by the method of Lowry et al. ${ }^{25)}$ using bovine serum albumin as a standard. The metabolism of SQV in liver or intestinal microsomes was measured in an NADPHgenerating system according to the following method. To a clean $15 \mathrm{ml}$ conical glass tube, $100 \mu \mathrm{l}$ of $5 \mathrm{~mm}$ NADPH in $0.1 \mathrm{M}$ phosphate buffer ( $\mathrm{pH} 7.4$ ), $100 \mu \mathrm{l}$ of $50 \mathrm{~mm}$ G6P in $0.1 \mathrm{M}$ phosphate buffer, $4 \mu \mathrm{l}$ of 500 units of G6PDH in $0.1 \mathrm{M}$ phosphate buffer, $100 \mu \mathrm{l}$ of $50 \mathrm{mM} \mathrm{MgCl}_{2}$ in $0.1 \mathrm{M}$ phosphate buffer, and $596 \mu \mathrm{l}$ of $0.1 \mathrm{M}$ phosphate buffer were added. In the inhibition experiment, $5 \mu \mathrm{l}$ of SQV ethanol solution was added. After a 5 -min preincubation at $37^{\circ} \mathrm{C}$ in a water bath, the metabolic reaction was initiated by adding $100 \mu \mathrm{l}$ of rat liver or intestinal microsomal suspension (the final concentration: 0.8 and $0.4 \mathrm{mg}$ protein $/ \mathrm{ml}$ of microsomal suspension, respectively) and incubated for $10 \mathrm{~min}$ at $37^{\circ} \mathrm{C}$. The reaction was stopped by the addition of $200 \mu \mathrm{l}$ of ice-cold $2 \mathrm{M} \mathrm{K}_{3} \mathrm{PO}_{4}$. The resultant mixture was used for the extraction to measure SQV concentration by the liquid chromatography-mass spectrometry (LC/MS). The initial metabolic reaction rate of PIs in rat intestinal microsomes was determined under linear conditions. Michaelis constant $\left(K_{\mathrm{m}}\right)$ and maximum reaction rate $\left(V_{\max }\right)$ were determined by Lineweaver-Burk plot analysis. $^{26)}$

Exsorption Study of Rho123 by in Situ Perfusion 
Method Rats were fasted for at least $12 \mathrm{~h}$ with free access to water and were anaesthetized with an intraperitoneal injection of sodium pentobarbital $(32 \mathrm{mg} / \mathrm{kg})$. The rats were placed in a supine position on a heating pad under a surgical lamp to maintain constant normal body temperature. A midline longitudinal abdominal incision was made, and the proximal end of the jejunum $(5 \mathrm{~cm}$ from the outlet of the bile duct) was catheterized with an inlet silicon tube $(4.2 \mathrm{~mm}$ i.d.), which was connected to a perfusion pump (Perista pump, Tokyo, Rikakikai Co., Ltd.). The distal end of the intestine $(5 \mathrm{~cm}$ from the ileocecum) was also cannulated with an outlet silicon tube to collect the intestinal effluent. Then, the lumen of the whole small intestine was flushed with $40 \mathrm{ml}$ D-PBS prewarmed at $37^{\circ} \mathrm{C}$. Subsequently, $5 \%$ ethanol D-PBS containing $25 \mathrm{~mm}$ glucose with different concentrations of IDV or $10 \mu \mathrm{M}$ CsA, a P-gp inhibitor, was perfused through the small intestine at a flow rate of $1 \mathrm{ml} / \mathrm{min}$ in a single perfusion. After $30 \mathrm{~min}$ of perfusion for stabilization, Rho123 solution $(100 \mu \mathrm{M})$ was injected at a bolus dose of $0.17 \mathrm{mg} / \mathrm{kg}$ via the jugular vein. Simultaneously, intestinal effluent perfusates were collected at 10-min intervals for $60 \mathrm{~min}$. Then, $0.25 \mathrm{ml}$ aliquots of blood samples were collected into heparinized centrifuging tubes at each midpoint of the intestinal effluent collection. The plasma samples were obtained by centrifuging the blood samples at $9000 \times \boldsymbol{g}$ for $10 \mathrm{~min}$. Control experiments were performed simultaneously in which IDV or CsA was omitted in the intestinal perfusate.

Exsorption Study of APV, SQV and NFV by in Situ Perfusion Method Exsorption of APV, SQV and NFV was determined in the absence or presence of $10 \mu \mathrm{M}$ IDV, whose concentration was determined from the above experiment, by perfusion method with rat small intestine in the manner described. APV, NFV or SQV solution was injected at a bolus dose of $5 \mathrm{mg} / \mathrm{kg}$ via the jugular vein. A control experiment was performed simultaneously in which IDV was omitted from the intestinal perfusate. Blood and perfusate samples were collected at designated times and were used for extraction immediately after the experiment.

Assay of Rho123 and HIV PIs The determination of Rho123 concentrations in the samples was performed immediately after the experiment. The perfuste and plasma samples were stored at $4{ }^{\circ} \mathrm{C}$ after collection. For plasma samples, an equal volume of methanol was added to $100 \mu \mathrm{l}$ aliquots of plasma for deproteinization. The mixture was vortex-mixed for $30 \mathrm{~s}$ and centrifuged at $12000 \times \boldsymbol{g}$ for $10 \mathrm{~min}$. Then, a 100 $\mu \mathrm{l}$ aliquot of the supernatant was added to the microplate and Rho123 concentrations in the samples were analyzed spectrofluorometrically with an excitation wavelength of $485 \mathrm{~nm}$ and an emission wavelength of $527 \mathrm{~nm}$. For perfusate samples, $100 \mu 1$ aliquot of each perfusate sample was added di- rectly to the microplate and Rho123 was analyzed as described above. The four HIV PIs were extracted with diethyl ether and measured using a previously described LC/MS analytical method with a negative APCI mode. ${ }^{27)}$

Data Analysis The values of area under the concentration versus time curve $(A U C)$ of Rho123 and HIV PIs after intravenous administration and other pharmacokinetic parameters were obtained by a non-compartmental pharmacokinetic analysis method to the plasma concentration-time data using the computer program, WinHARMONY. ${ }^{28)}$ The $A U C$ was calculated using the linear trapezoidal rule up to the last measured plasma concentration, $C p$ (last). The total body clearance $\left(C l_{\text {tot }}\right)$ was calculated by dividing an intravenous dose by $A U C$. The intestinal exsorption clearances $\left(C l_{\mathrm{ex}}\right)$ of Rho123 and HIV PIs were estimated by dividing the total exsorbed amount $\left(E x_{\text {tot }}\right)$ by the $A U C$. The metabolic clearance rate of each PI in the intestinal microsomes was calculated by dividing $V_{\max }$ by $K_{\mathrm{m}}$.

Statistical Analysis All values are expressed as the mean \pm S.D. Statistical comparisons were performed using one-way ANOVA followed by Tukey's multiple range test with a significance level of below 0.05 .

\section{RESULTS}

The effect of different concentrations of IDV and $10 \mu \mathrm{M}$ CsA in the perfusate on the exsorption of Rho123 from the blood to the intestinal lumen was studied, and the pharmacokinetic parameters of Rho123 are summarized in Table 1. In the presence of IDV, the total amount of Rho123 exsorbed from the bloodstream into the intestinal lumen, $E x_{\text {tot }}$, decreased as IDV concentration increased, indicating a concentration-dependent inhibition of Rho1 23 exsorption by IDV. In the presence of $10 \mu \mathrm{M}$ of IDV, the exsorbed percentage of Rho123 administered intravenously, estimated by dividing the total exsorbed amount by the intravenous dose, was quite close to that of CsA $(9.90 \pm 0.33 \%$ vs. $9.37 \pm 1.45 \%$ of the administered dose), and both were significantly lower than that of control in the absence of IDV in the intestinal perfusate $(p<0.05)$. The $C l_{\text {ex }}$ values of Rho123 also decreased in a concentration-dependent manner with IDV. In the presence of $10 \mu \mathrm{M}$ of IDV or CsA in the perfusate, $C l_{\mathrm{ex}}$ decreased significantly $(p<0.05)$, though the $A U C$ values of Rho123 showed no notable change. These observations suggested that IDV acted as an inhibitor of the P-gp, and that the inhibitory potency of $10 \mu \mathrm{M}$ IDV on the exsorption of Rho123 was comparable to that of $10 \mu \mathrm{M}$ CsA. Thus, $10 \mu \mathrm{M}$ IDV in the intestinal perfusate was chosen to investigate the effect of IDV on the intestinal exsorption of the other three PIs, APV, SQV and $\mathrm{NFV}$, following intravenous administration.

Table 1. Effect of IDV and CsA on the Exsorption of Rho123 after Intravenous Administration

\begin{tabular}{|c|c|c|c|c|c|}
\hline \multirow{2}{*}{ Parameter } & \multirow{2}{*}{ Control } & \multicolumn{3}{|c|}{ IDV $(\mu \mathrm{M})$} & \multirow{2}{*}{$\begin{array}{c}\mathrm{CsA}(\mu \mathrm{M}) \\
10\end{array}$} \\
\hline & & 0.1 & 1 & 10 & \\
\hline$A U C(\mu \mathrm{g} \cdot \mathrm{min} / \mathrm{ml})$ & $4.78 \pm 0.84$ & $4.17 \pm 0.13$ & $5.41 \pm 0.59$ & $5.77 \pm 1.60$ & $4.78 \pm 0.74$ \\
\hline$C l_{\mathrm{tot}}(\mathrm{ml} \cdot \mathrm{min})$ & $29.16 \pm 3.98$ & $30.60 \pm 0.53$ & $25.31 \pm 1.56$ & $25.89 \pm 4.13$ & $27.96 \pm 3.70$ \\
\hline$E x_{\text {tot }}(\mu \mathrm{g})$ & $8.44 \pm 1.17$ & $7.53 \pm 2.48$ & $5.41 \pm 0.59$ & $5.05 \pm 0.17 *$ & $4.78 \pm 0.74 *$ \\
\hline$C l_{\mathrm{ex}}(\mathrm{ml} / \mathrm{min})$ & $1.88 \pm 0.28$ & $2.16 \pm 0.79$ & $1.24 \pm 1.06$ & $1.06 \pm 0.17 *$ & $1.03 \pm 0.21 *$ \\
\hline
\end{tabular}

Each value represents the mean \pm S.D. of five rats. $* p<0.05$, compared with the control by ANOVA. 
Figure 2 shows the exsorption of APV, SQV and NFV from the blood circulation to the intestinal lumen after their intravenous administration in the absence or presence of $10 \mu \mathrm{M}$ IDV in the intestinal perfusate. The concentration of IDV in the plasma after perfusion reached around $0.35 \pm$ $0.081 \mu \mathrm{g} / \mathrm{ml}$, and this was lower than the inhibition constant against APV, SQV or NFV in the rat liver microsomes. ${ }^{13)}$ The pharmacokinetic parameters for APV, SQV and NFV are also listed in Table 2. The exsorbed amounts of SQV and NFV at an interval of $10-20 \mathrm{~min}$ increased significantly $(p<0.05)$. In the presence of IDV, there were no notable changes in the parameters; however, the mean values of $E x_{\text {tot }}$ and $C l_{\mathrm{ex}}$ tended to increase in comparison with the cases of Rho123.

To compare the metabolic clearance rates of the four HIV PIs via CYP enzymes in the intestine, their metabolism was studied with intestinal microsomal fractions. Table 3 shows the Michaelis constant $\left(K_{\mathrm{m}}\right)$, the maximum velocity $\left(V_{\max }\right)$ and the metabolic clearance rate $\left(V_{\max } / K_{\mathrm{m}}\right)$ of each of the four in the intestinal microsomes. The rank order of their metabolic clearance rates is $\mathrm{SQV}>\mathrm{NFV}>\mathrm{APV} \geqq \mathrm{IDV}$. Thus, IDV showed the smallest value in the metabolic clearance
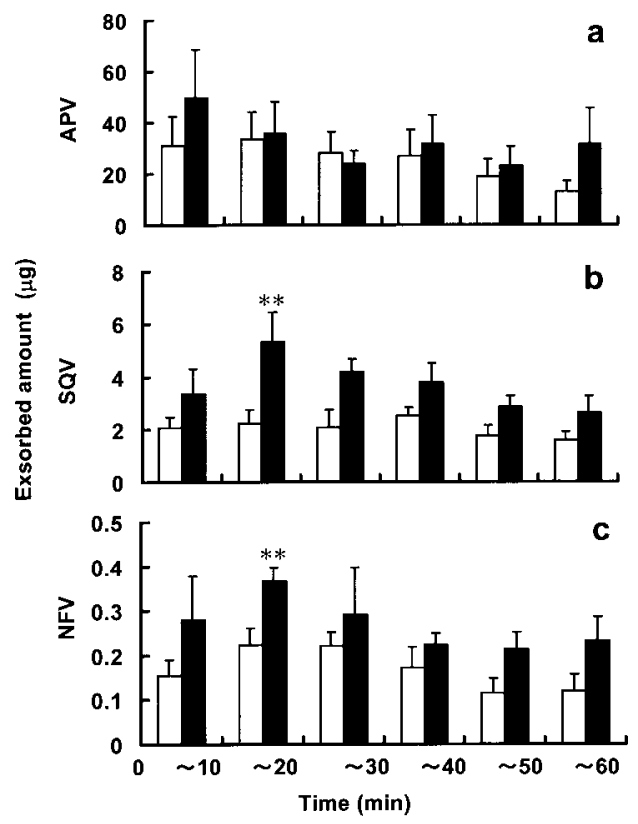

Fig. 2. Exsorption of APV (a), SQV (b) and NFV (c) Rho123 from the Bloodstream to the Intestinal Perfusate after Intravenous Administrations $(5 \mathrm{mg} / \mathrm{kg})$ in the Absence $(\square)$ and Presence ( $\square$ ) of $10 \mu \mathrm{M}$ IDV

IDV was passed through the entire small intestine at a flow rate of $1.0 \mathrm{ml} / \mathrm{min}$. The statistical significance of exsorbed amount of PIs in the absence or presence of IDV at each interval was evaluated against the same interval by ANOVA. The exsorbed amounts of SQV and NFV in the presence of IDV were significantly increased in comparison with those in the absence of IDV $(p<0.01)$. Each column with a bar represents the mean \pm S.D. of 6 rats. rate in the intestinal microsomes.

\section{DISCUSSION}

In recent years, the clinical management of patients infected with HIV has become more efficient through introduction of a highly-active antiretroviral therapy (HAART) composed of a combination of two kinds of reverse transcriptase inhibitors and an HIV PI. ${ }^{29)}$ However, many cases of treatment failure have been reported resulting from poor adherence to the therapy, development of viral resistance or pharmacokinetic reasons in HAART. ${ }^{30)}$ Recently, a combination therapy with two kinds of HIV PIs showed clinical effectiveness in preventing development of tolerance by HIV, and this double protease therapy has been attempted in clinical practice. ${ }^{1)}$ However, it is still difficult to choose an optimal combination of HIV PIs, because these drugs have considerable inter- and intra-patient variability in plasma concentrations and marked potential for drug interactions via CYP3A4. ${ }^{31,32)}$

In our previous studies on pharmacokinetic interactions among the HIV PIs currently used in clinical therapy, we observed that IDV inhibited the metabolism of APV, NFV and SQV with $K_{\mathrm{i}}$ values of $0.67,2.76$ and $3.55 \mu \mathrm{M}$, respectively. ${ }^{10-13)}$ After oral administration of APV, SQV or NFV with IDV, their $A U C$ values in the presence of IDV were increased 1.6-, 9.5- and 2.3-fold, respectively, compared with any of them alone. On the other hand, the $A U C$ values of APV, SQV and NFV after intravenous co-administration with IDV increased by 1.4-, 1.2- and 1.5-fold, respectively, and showed no notable increase compared to those obtained from oral administration. ${ }^{23}$ These observations clearly indicate that besides the liver, there is another site of pharmacokinetic interaction between IDV and the other three PIs. It is still true suspected that the small intestine is the possible site for those interactions among HIV PIs.

Our study on the hepatic and intestinal contribution to the pharmacokinetic interaction of IDV with other HIV PIs using a microdialysis technique demonstrated that the intestinal clearances of APV, SQV and NFV in combination with IDV

Table 3. Kinetics of Rat Intestinal Microsomal Metabolism of Protease Inhibitors

\begin{tabular}{cccc}
\hline \hline & $\begin{array}{c}K_{\mathrm{m}} \\
(\mu \mathrm{M})\end{array}$ & $\begin{array}{c}V_{\max } \\
(\mathrm{nmol} / \mathrm{min} / \mathrm{mg} \text { protein })\end{array}$ & $\begin{array}{c}V_{\max } / \mathrm{K}_{m} \\
(\mu \mathrm{l} / \mathrm{min} / \mathrm{mg} \text { protein })\end{array}$ \\
\hline APV & $7.03 \pm 3.05$ & $0.68 \pm 0.34$ & $90.39 \pm 7.18$ \\
NFV & $2.58 \pm 0.66$ & $0.60 \pm 0.10$ & $244.80 \pm 26.93$ \\
SQV & $3.73 \pm 1.59$ & $1.93 \pm 0.81$ & $523.16 \pm 4.50$ \\
IDV & $4.12 \pm 2.05$ & $0.32 \pm 0.18$ & $74.59 \pm 7.54$ \\
\hline
\end{tabular}

Each value represents the mean \pm S.D. of three determinations.

Table 2. Effect of IDV on the Exsorption of APV, SQV and NFV after Intravenous Administration

\begin{tabular}{|c|c|c|c|c|c|c|}
\hline \multirow{2}{*}{ Parameter } & \multicolumn{2}{|c|}{ APV } & \multicolumn{2}{|c|}{ SQV } & \multicolumn{2}{|c|}{ NFV } \\
\hline & Alone & $+\mathrm{IDV}$ & Alone & $+\mathrm{IDV}$ & Alone & $+\mathrm{IDV}$ \\
\hline$A U C(\mu \mathrm{g} \cdot \mathrm{min} / \mathrm{ml})$ & $79.88 \pm 6.74$ & $84.4 \pm 6.28$ & $66.19 \pm 24.90$ & $100.61 \pm 30.04$ & $58.44 \pm 9.03$ & $72.56 \pm 15.10$ \\
\hline$C l_{\text {tot }}(\mathrm{ml} / \mathrm{min})$ & $19.43 \pm 2.00$ & $18.75 \pm 1.79$ & $27.83 \pm 5.61$ & $19.74 \pm 5.63$ & $22.60 \pm 2.69$ & $17.31 \pm 2.65$ \\
\hline$E x_{\text {tot }}(\mu \mathrm{g})$ & $151.90 \pm 48.26$ & $189.94 \pm 55.67$ & $9.93 \pm 3.09$ & $14.58 \pm 3.22$ & $0.91 \pm 0.13$ & $1.38 \pm 0.20$ \\
\hline$C l_{\mathrm{ex}}(\mathrm{ml} / \mathrm{min})$ & $2.04 \pm 0.74$ & $2.39 \pm 0.77$ & $0.15 \pm 0.03$ & $0.21 \pm 0.08$ & $0.02 \pm 0.01$ & $0.02 \pm 0.01$ \\
\hline
\end{tabular}

Each value represents the mean \pm S.D. of five rats. 
were decreased by 32,53 , and $82 \%$, respectively, in comparison with that of any one of them alone, and also indicated that the extent of hepatic or intestinal contributions varied in each combination with IDV. $^{23)}$ The hepatic availabilities of APV, NFV and SQV after oral co-administration with IDV increased by 11,61 and $38 \%$, respectively, and the intestinal availabilities, which were expressed as a product of the fraction absorbed and the fraction unmetabolized, increased by 8 , 6 and 524\%, respectively. These observations suggested that the liver contributed to the increased bioavailabilities of NFV and SQV when they were orally co-administered with IDV, and that the intestine contributed more to the increase in SQV bioavailability. Therefore, both hepatic and intestinal metabolisms are essentially involved in the pharmacokinetic interactions between IDV and other HIV PIs.

It is well known that IDV, SQV, APV and NFV are substrates of both of P-gp and the CYP3A system. ${ }^{3,5,8,19)}$ Therefore, elimination of these drugs from the body through the intestine at least depends on the synergetic effect of CYP3A and P-gp function. In addition, the contribution of the P-gp efflux system into the intestine after oral administration of a drug is essentially given by the sum of transport of the drug from the bloodstream to the intestine and secretion of the drug just after being absorbed into the epithelial cells. However, it is unknown how these P-gp transport systems contribute to the interaction among HIV PIs, because no one has separately investigated the involvement of the P-gp transport system after oral administration. Accordingly, in the present study, we have focused on the role of P-gp in the effect of IDV on the exsorption of APV, SQV and NFV from the blood circulation to the intestinal lumen after intravenous administration using a single-pass perfusion system. In this system, the drug interaction between IDV and other HIV PIs in the liver was negligible, because the plasma concentration of IDV after perfusion reached around $0.35 \pm 0.081 \mu \mathrm{g} / \mathrm{ml}$, and this concentration range was lower than the inhibition constant against APV, SQV and NFV in the rat liver microsomes. ${ }^{13)}$ Since Rho123 is not a substrate of CYP3A, it would be quite suitable to use it as a marker to evaluate the in vivo P-gp function. ${ }^{33)}$ As shown in Table $1,10 \mu \mathrm{M}$ of IDV in the intestinal perfusate significantly decreased the values of $E x_{\text {tot }}$ and $C l_{\text {ex }}$ of Rho123. On the contrary, both $E x_{\text {tot }}$ and $C l_{\text {ex }}$ values of APV, SQV and NFV in the presence of IDV in the intestinal perfusate had an increasing tendency or remained unchanged, despite $10 \mu \mathrm{M}$ of IDV inhibiting the P-gp function. These observations clearly indicated that the mechanism of the interaction between IDV and other HIV PIs was different from that of IDV with Rho123.

In addition to the active efflux of drugs by the P-gp in the epithelial cells, the intestinal CYP enzymes also provide the first-pass metabolism for orally administered drugs. Indeed, a significant amount of CYP3A is expressed in the enterocytes to metabolize drugs during their transit across the intestinal epithelium, ${ }^{16,34)}$ and the CYP enzymes and P-gp in enterocytes might act synergistically in presystemic metabolism of HIV PIs. As listed in Table 3, IDV is hardly to be metabolized by CYP enzymes among the four HIV PIs. Judging from the magnitude of the metabolic clearance rates of PIs in the intestinal microsomes, it is assumed that SQV is most easily metabolically inhibited by IDV in the gut cells, which NFV following. These differences in metabolic inhibition by
IDV must be due to the differences in protein binding of PIs, because the free fraction of a drug is closely related to the intrinsic clearance or the first-pass metabolism. In our previous report, we found that the free fraction of APV, SQV, NFV and IDV in plasma was $0.02,0.15,0.06$ and 0.05 , respectively. ${ }^{12)}$ Therefore, the protein-binding ratio of IDV is larger than that of SQV or NFV, supporting the above phenomenon. In this study, although we focused on the exsorption of PIs via $\mathrm{P}$-gp from the bloodstream to the intestinal lumen after intravenous administration, it seems there is an additive effect by the inhibition of both CYP enzymes and the P-gp function. In the absence of IDV after intravenous administration, APV, SQV or NFV was taken up into the intestinal epithelial cells and some was by CYP3A or effluxed actively into the apical side by the P-gp in the cells. Drug molecules that escaped from metabolic conversion and active efflux were eliminated from the cells and sent into both the intestinal lumen and the blood circulation by a passive transport process. In the presence of IDV, P-gp was inhibited by IDV, thus the amount of the drugs effluxed into the apical side by P-gp decreased. Since IDV is also an inhibitor of CYP3A and all three PIs tested in this study are substrates of CYP3A, the metabolism of the HIV PIs in the enterocytes was also inhibited by IDV. This double inhibitory effect of IDV on the function of P-gp and CYP3A resulted in the increased amount of PIs inside the enterocytes, thus, enhancing the passive transport into both the intestinal lumen and the blood circulation. These mechanisms might be responsible for the fact that there was no notable change in the $C l_{\text {ex }}$ values of APV, SQV or NFV in the presence of IDV in comparison with those in the latter's absence.

In summary, we investigated the effect of IDV on the intestinal exsorption of other HIV PIs, i.e., APV, NFV and SQV from the blood-stream to the intestinal lumen after intravenous administration to rats. In the presence of IDV, the intestinal exsorption showed no notable change despite the fact that IDV inhibits the P-gp function nearly as much as CsA. The reason for this is due to the fact that IDV has an inhibitory effect on the intestinal metabolism of other PIs via CYP enzymes, and these effects are additive, accounting for the interactions between IDV and the other PIs. Of course, this does not mean the interaction between IDV and other PIs on the intestine is explained by these results alone. It is also necessary to determine the intestinal exsorption of PIs administered orally and to rank them in the order of their ability to inhibit P-gp or CYP3A in order to be able to discuss the intestinal interaction between HIV PIs.

\section{REFERENCES}

1) Barry M., Gibbons S., Back D., Mulcahy F., Clin. Pharmacokinet., 32, 194-209 (1997).

2) Finzi D., Blankson J., Siliciano D. J., Nat. Med., 5, 512-519 (1999).

3) Williams G. C., Sinko P. J., Adv. Drug Delivery Rev., 39, 211-238 (1999).

4) Caroline G. L., Gottesman M. M., Cardarelli C. O., Ramachandra M., Jeang K. T., Ambudkar S. V., Pastan R., Dey S., Biochemistry, 37, 3594-3601 (1998).

5) Choo E. F., Leake B., Wandel C., Imamura H., Wood A. J., Wilkinson G. R., Kim R. B., Drug Metab. Dispos., 28, 655-660 (2000).

6) Barry M., Mulcahy F., Merry C., Gibbons S., Back D., Clin. Pharmacokinet., 36, 289-304 (1999).

7) Chiba M., Hensleigh M., Lin J. H., Biochem. Pharmacol., 53, 1187 - 
1194 (1997).

8) Kim R. B., Fromm M. F., Wandel C., Leake B., Wood A. J., Roden D. M., Wilkinson G. R., J. Clin. Invest., 101, 289-294 (1998).

9) Lin J. H., Sugiyama Y., Awazu S., Hanano M., J. Pharmacokinet. Biopharm., 10, 649-661 (1982).

10) Yamaji H., Matsumura Y., Yoshikawa Y., Takada K., Biopharm. Drug Dispos., 20, 241-247 (1999).

11) Shibata N., Matsumura Y., Okamoto H., Kawaguchi Y., Ohtani A., Yoshikawa Y., Takada K., J. Pharm. Pharmacol., 52, 1239-1246 (2000).

12) Shibata N., Gao W., Okamoto H., Kishida T., Yoshikawa Y., Takada K., J. Pharm. Sci., 91, 680-689 (2002).

13) Shibata N., Gao W., Okamoto H., Kishida T., Yoshikawa Y., Takada K., J. Pharm. Pharmacol., 54, 221-229 (2002).

14) Kim J., Kim E. J., Han K. S., Chang M. S., Lee M. G., J. Pharm. Pharmacol., 51, 1031-1036 (1999).

15) Kim E. J., Han K. S., Lee M. G., Int. J. Pharmaceut., 194, 193-199 (2000).

16) Thummel K. E., O'Shea D., Paine M. F., Shen D. D., Kunze K. L., Perkins J. D., Wilkinson G. R., Clin. Pharmacol. Ther, 59, 491-502 (1996).

17) Tsuji A., Terasaki T., Takabakate Y., Tenda Y., Tamai I., Yamashima T., Moritani S., Tsuruo T., Yamasita J., Life Sci., 51, 1427-1437 (1992).

18) Terao T., Hisanaga E., Sai Y., Tamai I., Tsuji A., J. Pharm. Pharmacol., 48, 1083-1089 (1996).

19) Jerome H. H., Chiba M., Yamazaki M., Tang C., Lin J. H., J. Pharmacol. Exp. Ther, 298, 323-330 (2001).

20) Gottesman M., Pastan I., Annu. Rev. Biochem., 62, 385- 427 (1993).

21) Watcher V. J., Wu C. Y., Benet L. Z., Mol. Carcinogen., 13, 129-134 (1995).
22) Alsenz J., Steffen H., Alex R., Pharmaceut. Res., 15, 423-428 (1998).

23) Gao W., Kishida T., Kageyama M., Kimura K., Yoshikawa Y., Shibata N., Takada K., Antivir. Chem. Chemoth., 13, 35-44 (2002).

24) Fasco M. J., Silkworth J. B., Dunbar D. A., Kaminsky L. S., Mol. Pharmacol., 43, 226-232 (1993).

25) Lowry O. H., Rosebrough N. J., Farr A. L., Randall R. J., J. Biol. Chem., 193, 265-275 (1951).

26) Fitzsimmons M. E., Collins J. M., Drug Metab. Dispos., 25, 256-266 (1997).

27) Gao W., Kishida T., Kimura K., Kageyama M., Sumi M., Yoshikawa Y., Shibata N., Takada K., Biomed. Chromatogr., 16, 267-273 (2002).

28) Yoshikawa Y., Kato K., Sone H., Takada K., Jpn. J. Clin. Pharmacol. Ther., 29, 475-487 (1998).

29) Hoetelmans R. M., Reijers M. H., Weverling G. J., ten Kate R. W., Wit F. W., Mulder J. W., Weigel H. M., Frissen P. H., Roos M., Jurriaans S., Schuitemaker H., de Wolf F., Beijnen J. H., Lange J. M. A., AIDS, 12, F111-F115 (1998).

30) Vanhove G. F., Gries J. M., Verotta D., Sheiner L. B., Coombs R., Collier A. C., Blaschke T. F., Antimicro. Agents Chemother, 41, 24332488 (1997).

31) Hsu A., Granneman G. R., Cao G., Carothers L., Japour A., ElShourbagy T., Dennis S., Berg J., Erdoman K., Leonard J. M., Sun E., Antimicro. Agents Chemother., 42, 2784-2791 (1998).

32) Von Moltke L. L., Greenblatt D. J., Grassi J. M., Granda B. W., Duan S. M., Fogelmann J. P., Daily J. S., Harmatz Shader R. I., J. Clin. Pharmacol., 38, 106-111 (1998).

33) Yumoto R., Murakami T., Sanemasa M., Nasu R., Nagai J., Takano M., Drug Metab. Dispos., 29, 145-151 (2001).

34) Lin J. H., Chiba M., Chen I. W., Nishime J. A., Deluna F. A., Yamazaki M., Lin Y. J., Drug Metab. Dispos., 27, 1187-1193 (1999). 